\title{
Evaluation of the effectiveness of anti-turbulent additives in the oil pipeline transportation
}

\author{
Aleksei Balabukha, ${ }^{1, *}$, and Valentina Zvereva ${ }^{1}$ \\ ${ }^{1}$ Far Eastern Federal University, 690090, Vladivostok, Russia
}

\begin{abstract}
The authors of the article have developed the computer application allows to determine the value of the friction coefficient $\lambda$ and anti-turbulent additives efficiency with a high degree of accuracy. The program can be used in the calculations and design of oil pipelines. The paper presents experimental studies of the effect anti-turbulent additives on the magnitude of pressure losses during fluid movement through pipes. The data gained by the developed computer program has been proved by the data of practical application of additives in the real oil pipeline transportation system called Eastern Siberia-Pacific Ocean oil pipeline.
\end{abstract}

\section{Introduction}

The role of pipeline transportation for the modern economy is difficult to overestimate. Modern pipelines are tens, hundreds and thousands of kilometres long, transporting huge volumes of liquid. With the ever-increasing demand of the world economy for energy, an important task is to increase the throughput of oil pipelines. Until today, this problem has been solved by constructing larger pipelines, as well as by increasing the number and capacity of pumps, and, consequently, increasing the pressure at the outlet of the oil pumping stations. However, at the current stage of development of the oil pipeline transportation industry, limitations arise for this method. This occurs due to the need to ensure the safety in specific sections of the system, where restrictions on the pressure in the pipeline create a limit on the throughput of the entire pipeline [1,2].

Thus, today, one of the main tasks in the field of pipeline transportation of oil is increasing the throughput of the pipeline without increasing the pressure at the outlet of the pumping station. The use of so-called anti-turbulent additives today is becoming a mean of solving this problem. These additives are compounds of high molecular weight polymers, which, when introduced into the oil flow, lead to a decrease in turbulent eddies in the near wall layer of the flow. Due to this, the hydraulic resistance coefficient decreases and, therefore, while maintaining the pressure at the outlet of the oil pumping station, the oil throughput in the system increases $[3,4]$.

However, the use of such substances entails a number of related tasks to determine the optimal concentration of the additive, depending on the parameters of the system and antiturbulent agents. The nature of the effect of reducing the hydraulic resistance coefficient is complex. Therefore, the equations describing the influence of the concentration and

* Corresponding author: dein500@mail.ru 
properties of polymer additives on the fluid flow are often empirical and their solution requires the use of numerical calculation methods $[5,6]$.

In [7], the nature of the effect is considered, and various compounds are proposed as possible anti-turbulent agents. Both natural and synthetic polymeric compounds such as poly(ethylene oxide), poly(acrylic acid), polyacrylamide, poly(N-vinyl formamide) and gums were considered. The work also proposed some promising areas for the use of such substances.

In the study [8] the effect of degradation of molecule of polymer leading to a decrease in the turbulence reduction properties of additive is considered. As an alternative to polymeric agents, the use of surfactants that can act as anti-turbulent agents is proposed. Combinations of surfactants with a polyolefin were investigated for sensitivity to degradation when moving in a turbulent flow. It has been shown that such combinations reduce the wear of anti-turbulent agents.

The Ph.D Thesis [9] comprehensively addresses the issue of the movement of both a single-phase and a two-phase oil-water flow through tubes of various diameters. The main application of this research is primarily the possibility of increasing the coefficient of oil recovery from the field, since surfactants are currently actively used with this purpose in oil production. From the point of view of pipeline transportation of oil, the presented dependencies do not make it possible to carry out mathematical modeling of the process.

In [10], the effectiveness of the use of the modern anti-turbulent additive "MFLOWTREAT" brand C is considered. An experimental study of the effectiveness of the additive was conducted, depending on the decrease in pressure at the pump outlet and on the increase in fluid flow in the system. The experiment was carried out in four operating modes with additive concentration of $0,10,20,30 \mathrm{ppm}$. The results of studies have shown the feasibility of using anti-turbulent agents. However, the problem of mathematical modeling for calculating the most effective concentration of the additive depending on the parameters of the system and the pipeline has not been solved.

The problem of mathematical modeling of the process of oil pipeline transportation with use of anti-turbulent additives is considered in [11]. The effectiveness of modern additives was studied depending on their concentration. As the mathematical model, the system of nonlinear equations was proposed that were solved using the Newton-Raphson numerical method. The dependences of the efficiency coefficient on the additive concentration were derived. However, the presented equations are derived for the considered types of additives and are not universal.

\section{Theoretical basis}

The effect originates in the layers of the fluid flow near the pipe wall, where the so-called lamination of the fluid particles movement occurs, i.e., a decrease in the turbulence of the wall layer. This leads to an increase in the throughput of the pipeline at a constant pumps pressure or to a decrease in the power consumption of the pumps while maintaining the initial pipeline performance. The mechanism of this effect is shown in Figure 1 [12]. 


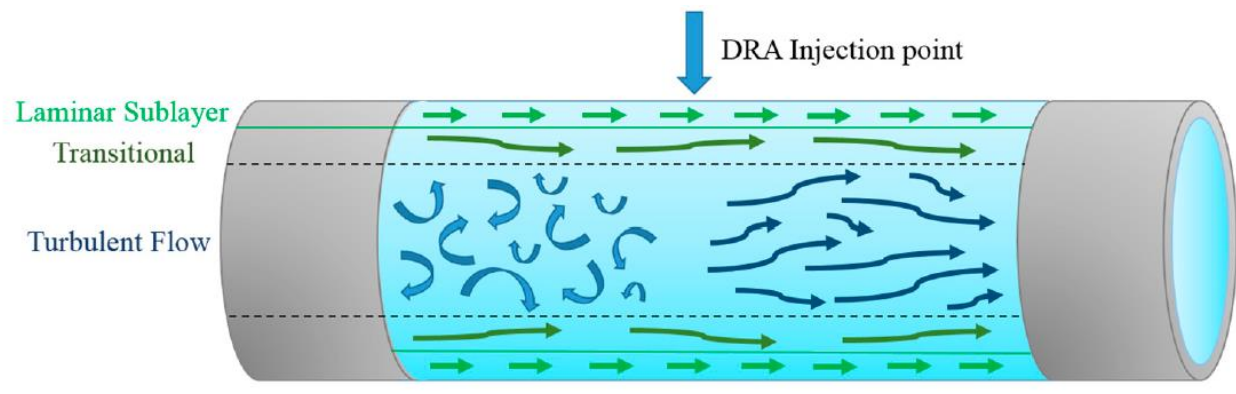

Fig. 1. Schematic representation of the effect of reducing flow turbulence with the introduction of polymer additives [12].

There are various theories that explain the nature of the processes that occur when polymer additives are introduced into a fluid stream:

- Orientation of liquid molecules around the macromolecules of polymers with the formation of structures that reduce the hydraulic resistance inside the flow due to the extinction of turbulent eddies.

- Adsorption of polymer molecules by the walls of the pipeline and smoothing of wall roughness with the creation of a film.

- Giving the properties of non-Newtonian fluids to solutions of high molecular weight polymers, such as pseudoplasticity. The effective viscosity of such fluids decreases with increasing pumping volumes. In addition, the concentration of anti-turbulent polymeric agents is millionths of the total volume of the solution. Therefore, interaction of polymer macromolecules is not manifested. However, if concertation of active component is increased sticking together can occur avoided leading to a reduction of the effectiveness of such substances [13].

The effectiveness of the use of anti-turbulent additives is determined by the molecular weight of the active polymer and the operating parameters of the pipeline (flow rate, pipeline diameter, temperature, viscosity of the pumped oil, etc.). In the case of the use of anti-turbulent additives in pipelines, the problem arises of determining its efficiency, which is calculated by the formula (1)

$$
\psi=\frac{\lambda_{0}-\lambda_{f}}{\lambda_{0}} \cdot 100 \%=\left(1-\frac{\Delta P_{f} \cdot Q_{0}^{2}}{\Delta P_{0} \cdot Q_{f}^{2}}\right) \cdot 100 \%
$$

where $\lambda_{f}, \lambda_{0}$ are the coefficients of hydraulic resistance during oil flow with and without additive, $\Delta P_{f}, \Delta P_{0}$ - pressure loss during oil flow with and without additive, $Q_{f}, Q_{0}-$ oil consumption with and without additive [14].

Thus, the effectiveness of the anti-turbulent additive is determined after its application in a certain section of the pipeline, depending on the increase in productivity and / or decrease in pressure at the outlet of the pump station.

Modern polymer additives must primarily meet the requirements of economic efficiency. Therefore, different anti-turbulent additives are used in different countries. But in all cases, the leaders in this area are specialized solutions of macromolecular compounds with a linear molecular structure. For example, brands FLO-XL, FLO-MXA, MFLOWTREAT, PT-Flyde, etc. In such additives, the content of the active polymer reaches $25 \%[15]$.

The effect of polymer additives on the decrease in the hydraulic resistance coefficient $\lambda$ was investigated in $[16,17]$. According to the data of experiments with the movement of 
water in pipelines with the addition of polymers, the hydraulic resistance coefficient $\lambda$ under the turbulent regime of fluid motion can be calculated by the formula

$$
\frac{1}{\sqrt{\lambda}}=-2 \lg \left[\left(\frac{2.8 V^{* 2} c}{V \sqrt{\lambda}}\right)^{\beta / 5,75}\left(\frac{2.51}{\operatorname{Re} \sqrt{\lambda}}+\frac{\Delta e}{3,701}\right)\right],
$$

where $V^{*}{ }_{c}$ is the threshold dynamic velocity $(\mathrm{m} / \mathrm{s})$ at which the decrease in pressure loss begins, $\beta$ is the coefficient depending on the type of polymer and its concentration.

For example, for polyacrylamide, it is recommended to take $V^{*}{ }_{c}=0.05 \mathrm{~m} / \mathrm{s}$, and $\beta$ at $(0.005 \%<\mathrm{C}<0.012 \%)$ calculate by the empirical formula

$$
\beta=1000 \mathrm{C},
$$

where $\mathrm{C}$ is the volumetric concentration of the polymer (\%).

When $C=0 ; \beta=0$, i.e., in the absence of a polymer additive, equation (2) is transformed into the well-known Kolbrook - White formula

$$
\frac{1}{\sqrt{\lambda}}=-2 \lg \left(\frac{2.51}{R e \sqrt{\lambda}}+\frac{\Delta e}{3.701}\right),
$$

i.e. one of the equations of hydraulics for determining $\lambda$ during fluid motion without polymer additives.

Equation (2) makes it possible to include both the parameters of the pipeline system and the polymer additive parameters in the calculation. Therefore, it is promising for calculating the optimal concentration of the additive. However, the calculation according to this equation is complicated by the fact that, due to its empirical nature, the solution of equation (2) involves enumerating a large number of values.

\section{Purpose and objects of research}

It is suggested that the main solution to the mentioned problem is the creation of the specialized computer program for calculating the effectiveness of polymer additives depending on the parameters of the pipeline, the pumped oil and the parameters of the antiturbulent additive. Equation (2) was chosen as the mathematical dependence underlying the developed program. It was decided that the most convenient program would be the program in the form of an application that can be installed on any computer. The developed program was created in the Python and is designed for all modern operating systems.

Input data:

- Equivalent roughness of a pipe $(\Delta \mathrm{e}[\mathrm{mm}])$

- The inner diameter of the pipe $(\mathrm{d}[\mathrm{m}])$;

- The flow rate of the fluid in the pipe (Q $[\mathrm{m} 3 / \mathrm{s}])$;

- Kinematic viscosity of the medium (V $[\mathrm{m} 2 / \mathrm{s}])$;

- Threshold speed (tabular value for the selected additive, $\left(\mathrm{V}_{-} \mathrm{c}[\mathrm{m} / \mathrm{s}]\right)$ );

- Volumetric concentration of the polymer additive $(\mathrm{C}[\%])$.

The result is the calculated value of the friction coefficient $\lambda$ of the polymer additive solution depending on the concentration of the polymer additive in solution. 


\section{Development of application}

Consider equation (2). It is required to find $\lambda$ for other known quantities. Note that the lefthand side of the equation is a decreasing function of a positive sign. The right-hand side is an increasing function. We will write it in more detail.

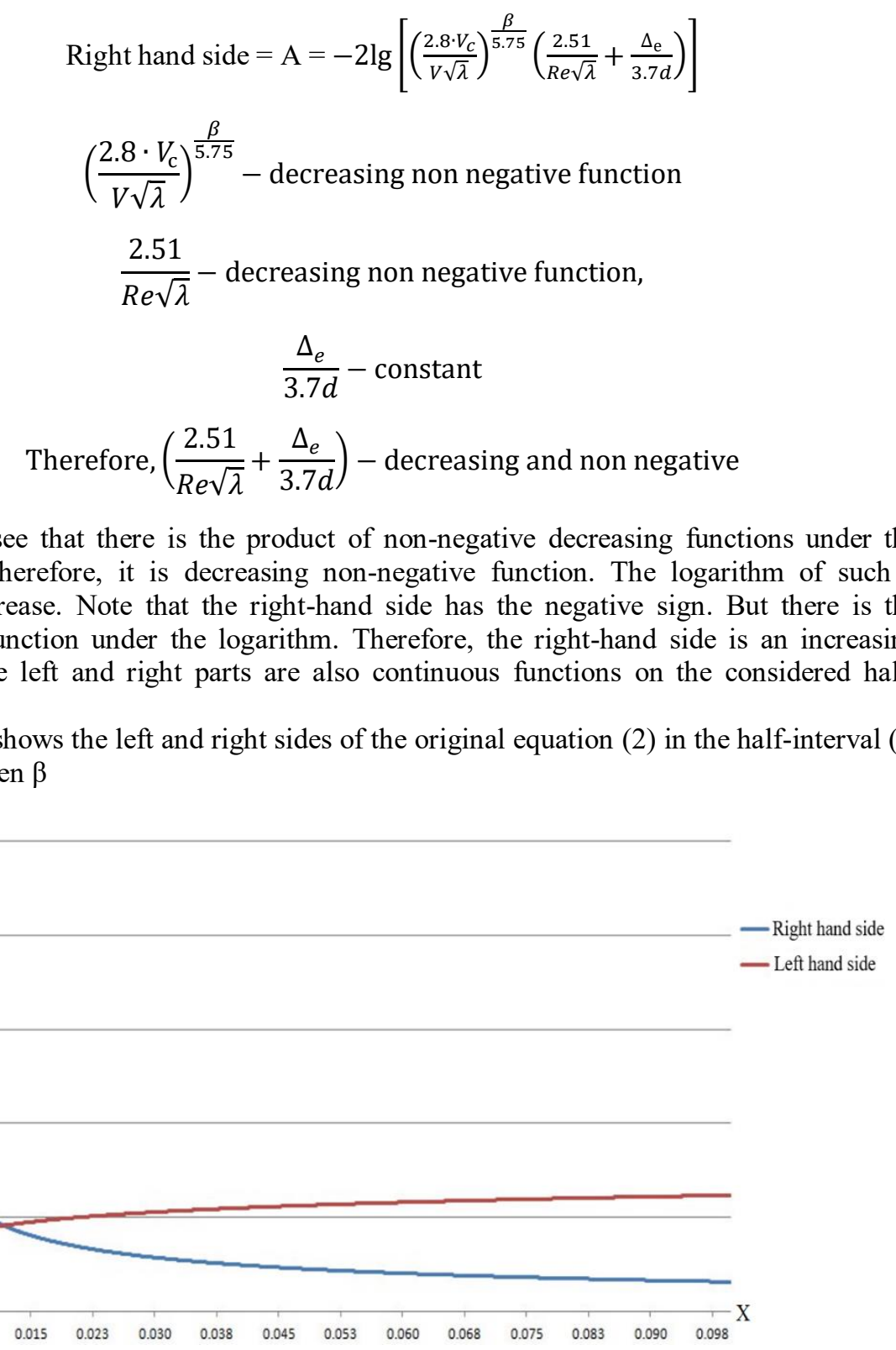

Fig. 2. The left and right sides of the equation (2) in the half-interval $(0,0.1)$ for a given $\beta$.

Define the function of discrepancy as the difference between the left and right sides. 


$$
f(\lambda)=\frac{1}{\sqrt{\lambda}}+2 \lg \left[\left(\frac{2.8 \cdot v_{c}}{V \sqrt{\lambda}}\right)^{\frac{\beta}{5.75}}\left(\frac{2.51}{\operatorname{Re} \sqrt{\lambda}}+\frac{\Delta_{e}}{3.7 d}\right)\right], \lambda \in[a, b]
$$

We get the following graph - Figure 3 .

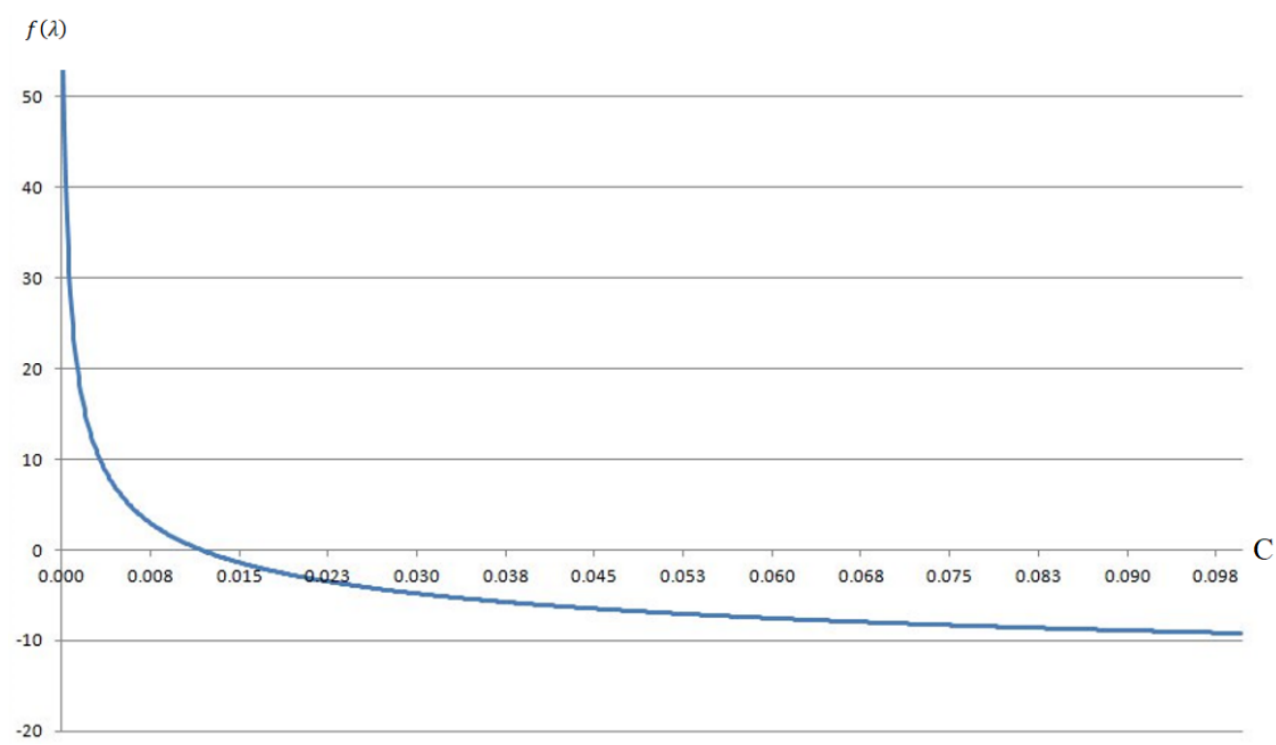

Fig. 3. Discrepancy function.

We are looking for a solution on the half-interval $(0,1]$. We see that on the left boundary of the half-interval the function takes a positive value, while on the right boundary it is negative. The desired point is the zero of the discrepancy function. To solve this, we apply the Dichotomy method.

Thus, in spite of the fact that this problem is difficult to solve analytically, it can be solved numerically with any predetermined accuracy.

Equations (2) and (3) are developed on the basis of experiments with polyacrylamide. For this reason, it becomes necessary to supplement these equations to calculate the effectiveness of other anti-turbulent additives. It was decided to introduce a new empirical coefficient into the initial dependence. Thus, equation (2) takes the following form.

$$
\frac{1}{\sqrt{\lambda}}=-2 \lg \left[\left(\frac{2.8 V^{* 2}{ }_{n o p}}{V \sqrt{\lambda}}\right)^{Z \cdot C / 5.75}\left(\frac{2.51}{R e \sqrt{\lambda}}+\frac{\Delta e}{3.701}\right)\right],
$$

where $\mathrm{Z}$ is the empirical coefficient of the influence of the concentration of the additive on the viscosity of the solution. This parameter depends on the type of additive selected.

In equation (3), which was discussed above, this coefficient was equal to 1000. This parameter was determined for polyacrylamide. For modern anti-turbulent additives, this coefficient can be equal to 10,000 . This indicates a more significant effect of polymers with a higher molecular weight on the viscosity of the solution.

The program was written in the Python development environment. It includes a user interface and allows user to calculate performance curves for various anti-turbulent additives. In addition, the application displays the calculated points of the graph in a separate file. Thanks to this, the user can process the data of a computer experiment. The developed program in the form of an application for a personal computer received a 
certificate of registration of a computer program [18]. Figure 4 shows the interface of the developed program and the main parameters of the pipeline and anti-turbulent additive.

$$
\frac{1}{\lambda}=-2 \log _{10}\left[\left(\frac{2.8 v_{c}^{*}}{v \sqrt{\lambda}}\right)^{\beta / 5.75}\left(\frac{2.51}{R e \sqrt{\lambda}}+\frac{\Delta_{e}}{3.7 d}\right)\right]
$$

$\lambda$ - hydraulic friction coefficient

$V_{c}^{*}$ - critical value of flow speed

$V$ - mean flow speed

$R e$ - Reynolds number

$d$ - pipeline diameter

$\Delta_{\mathbf{e}}$ - roughness of pipeline wall

$\beta=Z^{*} C$, where $C$ - volumetric concentration of additive

\section{Pipeline parameters}

roughness of pipeline wall, $\mathrm{mm}$

mean flowrate, $\mathrm{m}^{\wedge} 3 / \mathrm{c}$

pipeline diameter, $\mathrm{mm}$

oil kinematic viscosity, cSt

\begin{tabular}{|l|}
\hline 0.1 \\
\hline 1.425 \\
\hline 1000 \\
\hline 12.2
\end{tabular}

\section{Additive parameters}

additive kinematic viscosity, $\mathrm{cSt}$

critical flow speed, $\mathrm{m} / \mathrm{s}$

$\mathrm{Z}$ koefficient

\begin{tabular}{|l|l|}
\hline 1700 \\
\hline 0.04 \\
\hline 10000 \\
\hline
\end{tabular}

\section{Graph parameters}

min. additive concentration, ppm

max additive concentration, ppm

number of point in graph

\begin{tabular}{|l|}
\hline 0 \\
\hline 350 \\
\hline 30 \\
\hline
\end{tabular}

Create graph

Fig. 4. Interface of developed computer application.

\section{Calculation of the effectiveness of modern anti-turbulent additives}

The calculations in the presented work were carried out both with the help of the developed software, and with the help of existing methods for assessing the effectiveness of polymer additives by known operational characteristics. To calculate effectiveness of an additive the following data are required. Pressure reduction at the outlet of the oil pumping station and growth of the throughput in the pipeline section.

Making changes to the program code allowed to expand the functionality of the project. Figure 5 shows the calculation of the effectiveness of the FLO-MX anti-turbulent additive. 


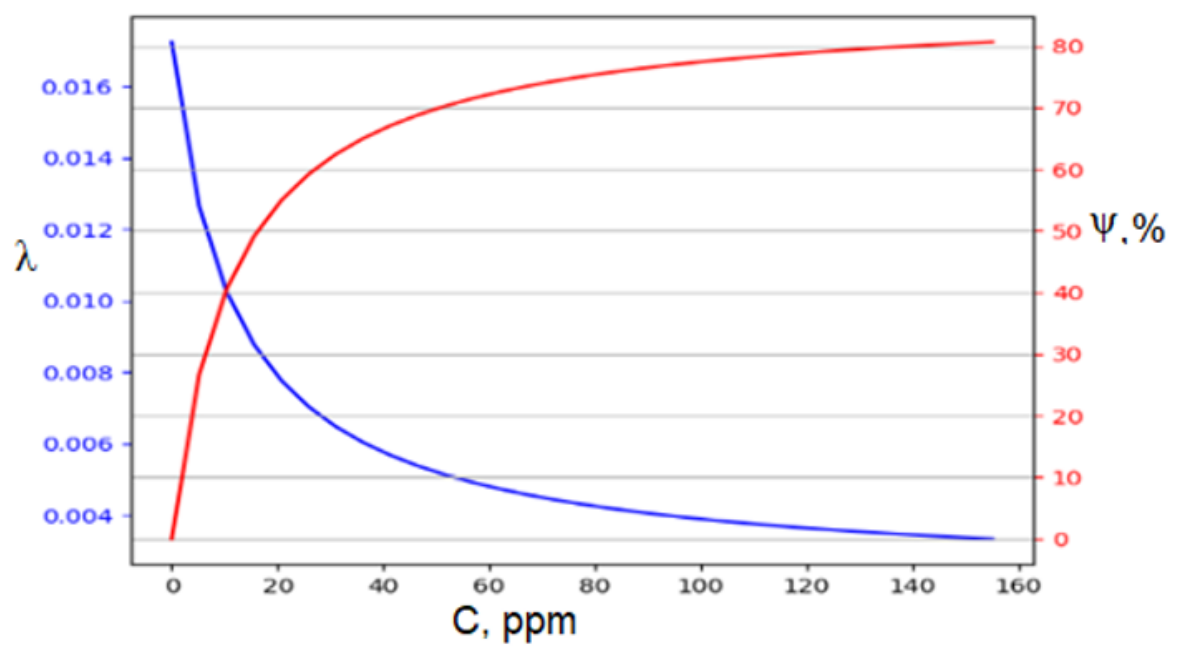

Fig 5. Graph of FLO-MX efficiency versus concentration.

To compare the results obtained by the developed program the manufacturer data was used. Figure 6 presents the data of Baker Hughes company on the effectiveness this type of anti-turbulent additive [19].

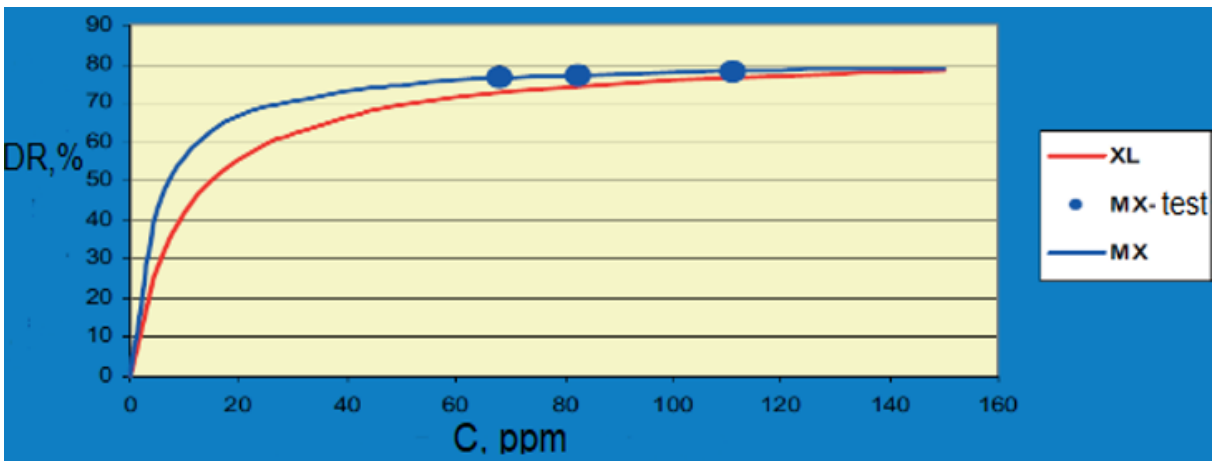

Fig. 6. Graph of FLO-MX efficiency versus concentration shown by manufacturer [19].

As can be seen from the graphs, the results gained by the developed program confirm the experimental values provided by the manufacturer company in the entire area of effective concentration.

In the further work, the task was set to assess the decrease in the efficiency of additive along the pipeline length with parameters corresponding to the Eastern Siberia - Pacific Ocean oil pipeline system. The limiting section of the pipeline was considered in two operating modes with the use of an anti-turbulent additive and without it [20]. Table 1 presents the operational parameters of the section with additive. Table 2 presents the similar parameters of the section when it is operated without the use of anti-turbulent additive. As can be seen from the data in tables 1 and 2, oil flowrate in the pipeline section from Station 1 to Station 2 increased by $2 \%$ with the introduction of additive. In addition, with constant pressure at the end of the pipeline section, it was possible to reduce the pressure at the outlet of pump station 1 by $3.2 \%$. Therefore, the total effectiveness of additive in the selected section was $7 \%$ according to the formula (1) 
Table 1. Typical data of Pressure and Flowrate of oil pipeline section using PT-Flyde anti-turbulent additive with a flow rate of additive used as 12 liters per hour, which is equal to $2.1 \mathrm{ppm}$

\begin{tabular}{|c|c|c|c|c|c|}
\hline & $\begin{array}{c}\text { Pressure, } \\
\mathrm{MPa}\end{array}$ & Length, $\mathrm{km}$ & $\begin{array}{c}\text { Elevation, } \\
\mathrm{m}\end{array}$ & $\begin{array}{c}\text { Oil Flowrate, } \\
\mathrm{m}^{3} / \mathrm{h}\end{array}$ & $\begin{array}{c}\text { Additive } \\
\text { concentration, } \mathrm{ppm}\end{array}$ \\
\hline Station-1 & 6.13 & 0 & 253.7 & \multirow{2}{*}{5211.19} & 2.1 \\
\cline { 1 - 4 } Station-2 & 0.67 & 259.6 & 369.5 & & 2.19 \\
\hline
\end{tabular}

Table 2. Typical data of Pressure and Flowrate of oil pipeline section without using the anti-turbulent additive

\begin{tabular}{|c|c|c|c|c|}
\hline & Pressure, MPa & Length, $\mathrm{km}$ & Elevation, $\mathrm{m}$ & $\begin{array}{c}\text { Oil Flowrate, } \\
\mathrm{m}^{3} / \mathrm{h}\end{array}$ \\
\hline Station-1 & 6.33 & 0 & 253.7 & \multirow{2}{*}{5116.69} \\
\cline { 1 - 4 } Station-2 & 0.67 & 259.6 & 369.5 & \\
\hline
\end{tabular}

We will analyze the average efficiency of the PT-FFLYDE additive taking into account the main parameters of the pipeline section using the developed program (Figure 7).

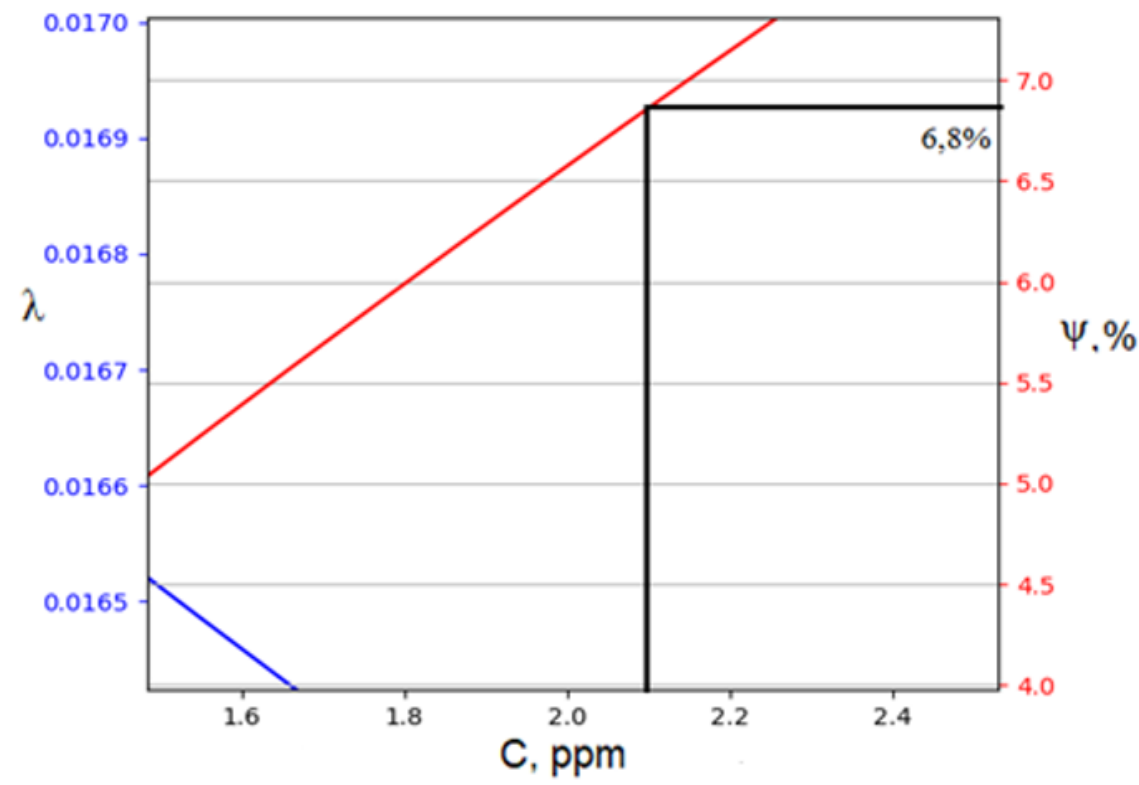

Fig. 7. Calculation of the PT-FLYDE additive effectiveness for the selected section of the pipeline using the developed computer application

When calculating the efficiency of the additive used for the oil pipeline section, the average efficiency value of $6.8 \%$ was obtained, which meets the requirements of calculation accuracy using analytical tools.

\section{Conclusion}

On the basis of practical experiments conducted in the laboratory, as well as studies of theoretical aspects of effectiveness of anti-turbulent additives evaluation in the oil pipeline 
transport, the following main results were obtained that determine the practical value of the work performed.

As a result of theoretical studies of the scientific literature on the use of polymer additives, the main solutions to improve the energy efficiency of oil pipeline transport were considered. A comparative analysis was carried out, on the basis of which the main limiting factors were identified, as well as the most promising technologies for increasing the capacity of main oil pipelines.

A new empirical coefficient is proposed for the dependency, which allows calculating the hydraulic resistance coefficient depending on the parameters of the pipeline system and the parameters of the polymer additive. This made it possible to refine the calculation of the effectiveness of anti-turbulent additives over the entire area of effective concentration.

A software product has been developed. It allows with, a high degree of accuracy, calculating the effectiveness of anti-turbulent depending on the parameters of the pipeline and the parameters of the polymer additive.

\section{References}

1. C.A. de Sousa, O.J. Romero, Latin American Journal of Energy Research 4(1), 17-29 (2017). doi: 10.21712/lajer.2017.v4.n1.p17-29

2. E.B. Priyanka, C. Maheswari, B. Meenakshipriya, Journal of Applied Research and Technology 14, 125-131 (2016). http://dx.doi.org/10.1016/j.jart.2016.03.004

3. A. Japper-Jaafar, M. B. Escudier, R. J. Poole, Journal Non-Newtonian Fluid Mechanics 161, 86-93 (2010).

4. V.N. Manzhai, M.M.C. Le Grand, A.V. Abdousaliamov, IOP Conf. Series: Earth and Environmental Science 21, 012028 (2014), doi:10.1088/1755-1315/21/1/012028.

5. A. Abubakar, A. Al-Hashmi, T. Al-Wahaibi, Y. Al-Wahaibi, A. Al-Ajmi, M. Eshrati, Adv. Mech. Eng. 6, 202073 (2014).

6. H.R. Karami, D. Mowla, J. Nonnewton. Fluid Mech. 177-178, 37-45 (2012).

7. W.J. Han, Y.Z. Dong, H.J. Choi, Processes 5(2), 24 (2017). doi:10.3390/pr5020024

8. X. Dai, G. Li, J. Li, Y. Xin, G. Yang, Y. Zhang, 2nd International Workshop on Materials Engineering and Computer Sciences (2015).

9. L.C. Edomwonyi-Otu, Drag reduction in oil-water flows, Ph.D Thesis (Department of Chemical Engineering University College London, 2015).

10. A. K. Nikolaev, N. A. Zaripova, A. A. Erashov, E. S. Demenin, IOP Conf. Series: Earth and Environmental Science 194, 082029 (2018). doi:10.1088/17551315/194/8/082029

11. M. Bogdevičius, J. Janutėnienė, K. Jonikas, E. Guseinovienė, M. Drakšas, Journal of Vibroengineering 15(1) 419-427 (2013).

12. W.J. Han, H.J. Choi, Polymers 9, 209 (2017). doi:10.3390/polym9060209

13. G. Yunqing, L. Tao, M. Jiegang, S. Zhengzan, Z. Peijian, An Applied Bionics and Biomechanics 6858720 (2017). https://doi.org/10.1155/2017/6858720

14. B. A. Jubran, Y. H. Zurigat, M. F. A. Goosen, Petroleum Science and Technology, 23, 1403-1424 (2005). DOI: 10.1081/LFT-200038223

15. C.H. Hong, C.H. Jang, H.J. Choi, Polymers 7, 1279-1298 (2015). doi:10.3390/polym7071279

16. L. Xi, Physics of Fluids 31, 121302 (2019); https://doi.org/10.1063/1.5129619 
17. C.M. White, M.G. Mungal, Annual Review of Fluid Mechanics 40, 235-256 (2008). https://doi.org/10.1146/annurev.fluid.40.111406.102156

18. A. Kalinina, L. Pushkareva, A. Rybakova, Amazonia Investiga 8(22), 40-50 (2019).

19. FLO Baker Hughes Anti-Turbulent Additives Company Website, https://www.bakerhughes.com/turbulent+additives

20. P. A. Revel-Muroz, Y. M. Fridlyand, S. E. Kutukov and A. I. Golyanov, Pipeline Science and Technology 3(1), 4-14 (2019). 